\title{
Valores y actitudes de los/as jóvenes andaluces en contextos pluriculturales $^{1}$
}

\author{
María del Rocío Cárdenas Rodríguez \\ Universidad Pablo de Olavide
}

\section{Resumen}

Uno de los grandes desafíos actuales lo constituye el conocimiento de las nuevas generaciones. El mundo de los valores de los jóvenes resulta, por muchas razones, muy desconocido. El pensamiento de los jóvenes con respecto a la sociedad en la que viven y la convivencia con otras culturas resulta ser un interrogante. Pero un interrogante sobre el que deseamos y necesitamos tener información. Lo deseamos porque los jóvenes son una de las partes más importantes de nuestra estructura social, y lo necesitamos porque, sin ese conocimiento, resulta difícil planificar el propio futuro sobre las bases de una convivencia democrática.

Palabras Clave: Valores, Diversidad cultural, Inmigrantes, Convivencia, Interculturalidad.

\section{Summary}

One of the great present challenges constitutes the knowledge of the new generations. The world of the values of the young people is, for many reasons, very not known. The thought of the young people with respect to the society in whom they live and the coexistence with other cultures turns out to be a question. But a question on which we wished and we needed to have information. We wished it because the young people are one of the most important parts of our social structure, and we needed it because, without that knowledge, she is difficult to plan the own future on the bases of a democratic coexistence.

KEYWORDS: Values, Cultural diversity, Immigrants, Coexistence, Interculturality.

\section{Introducción}

Debido a la amplitud de los movimientos migratorios provocados por los problemas económicos y sociales en países en desarrollo y por los conflictos bélicos, las sociedades son cada vez más multiculturales, conformadas por diversos grupos étnicos y por colectivos culturalmente diferentes.

Aunque en menor medida, el desarrollo de la sociedad española no es diferente del resto de las sociedades europeas y cada vez más existe una multiplicidad de culturas den- 
tro del territorio español. Como consecuencia, nos encontramos inmersos en una realidad diferente a la de hace tan sólo unas décadas; así, de un país emisor de emigrantes hemos pasado a constituir un país de destino de inmigrantes. El crecimiento económico y social que ha sufrido España en los últimos años, así como su situación geográfica, han influenciado en acelerar esta transformación.

El presente estudio, realizado en el año 2005, pretende conocer los valores y actitudes dominantes entre los/as jóvenes andaluces, así como las percepciones que tienen del fenómeno migratorio.

La investigación que a continuación presentamos tiene como fin primordial establecer las acciones pertinentes para convivir en una sociedad integrada, acciones que deben desarrollarse en los dos agentes principales del presente estudio: el colectivo de inmigrantes y el colectivo autóctono.

Lo que está en juego no son más ni menos que las relaciones humanas, nuestra capacidad de vivir juntos en el respeto a la libertad ajena. Para ello es necesario crear conciencia en los/as jóvenes de sus potencialidades y despertarles el deseo de mejorar el entorno social, puesto que en una democracia todos somos responsables de la dinámica de la sociedad.

Esta investigación parte de una serie de objetivos:

- Identificar la percepción, estereotipos y actitudes que tienen los/as jóvenes andaluces ante diferentes colectivos y el grado de tolerancia predominante entre los/as jóvenes andaluces. - Determinar la creencia de los/as jóvenes andaluces en los valores democráticos de la sociedad civil en la que se desarrollan como ciudadanos.

- Establecer el conocimiento que tienen los/as jóvenes andaluces sobre el fenómeno migratorio y su repercusión en la sociedad.

- Comparar los resultados obtenidos en esta investigación con otra investigación realizada a nivel nacional.

\section{Metodología}

La primera etapa del método científico es la identificación del problema objeto de estudio. En este sentido, y siguiendo a Pérez de Guzmán Puya (2003), el objetivo de nuestra investigación no es conocer, nuestra finalidad radica en "saber, saber ser y saber hacer para vivir y convivir. El objetivo es comprender realidades, no juzgarlas".

En nuestro caso partimos de una revisión bibliográfica sobre el tema que nos ocupa, así como de la experiencia previa en esta línea de trabajo. El problema general que nos interesa es el siguiente: ¿qué prejuicios y estereotipos presentan los/las jóvenes andaluces ante diferentes culturas, razas y grupos sociales marginales? ¿Cuál es el conocimiento de los/as jóvenes andaluces sobre la realidad del fenómeno migratorio? ¿Creen los/as jóvenes andaluces en las bases democráticas de la sociedad civil que establecen la igualdad de derechos y obligaciones de todas las personas?

Para poder dar respuesta a estos interrogantes partimos de un paradigma cuantitativo. El paradigma seleccionado condiciona los procedimientos de estudio que sigue una investigación, pues cada paradigma mantiene una concepción diferente de lo que es la investigación, cómo investigar, qué investigar y para qué sirve la investigación.

En el marco inductivo de esta investigación hemos utilizado la encuesta como método para indagar el conocimiento de la realidad de los/as jóvenes. La información resultante de las variables del estudio permite que realicemos las inferencias y las generalizaciones pertinentes. El método de encuesta en esta circunstancia resulta muy adecuado porque permite recoger datos a los que de otra forma sería muy difícil acceder, si no imposible de conseguir.

En esta investigación podemos señalar las siguientes variables a través de las cuales se analizan las percepciones y opiniones de 
los/as jóvenes andaluces sobre determinados colectivos y grupos sociales que representan alguna diferencia importante con respecto a su propia cultura, así como el conocimiento que tienen del fenómeno de la inmigración:

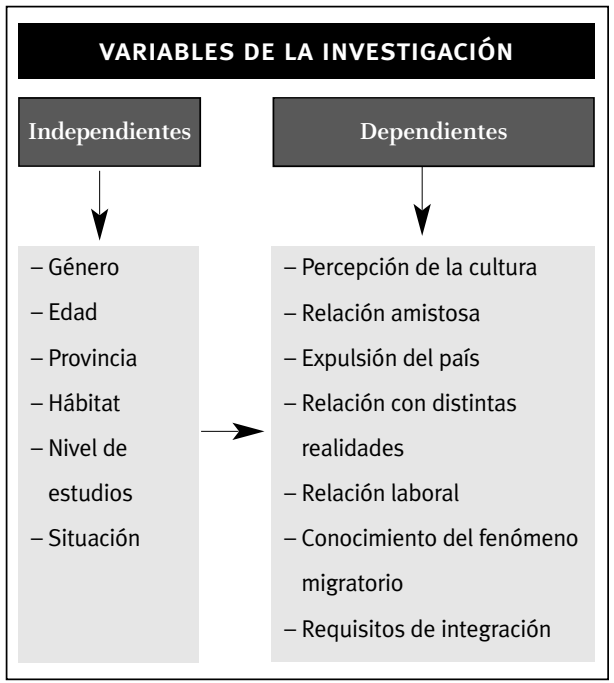

Para llevar a cabo la recogida de información hemos elaborado un cuestionario a partir de otros instrumentos ya utilizados y de otros cuestionarios elaborados para diversos estudios centrados en problemáticas semejantes a las planteadas. Nosotros hemos puntualizado y añadido cuestiones para recoger determinada información.

Para calcular la fiabilidad se ha utilizado el método de consistencia interna, el cual nos permite establecer la fiabilidad del instrumento sin elaborar otras formas o pasarlo más de una vez. Aplicamos el coeficiente alfa de Cronbach, el cual se adapta a nuestro cuestionario. Para su cálculo empleamos la técnica del coeficiente alfa estandarizado, cuyo cálculo se basa en la media de las correlaciones entre todos los ítems. De este modo se obtiene el coeficiente total de fiabilidad de la prueba y los de cada ítem con el total.

El estudio de la validez de nuestro instrumento se ha realizado mediante la evaluación de expertos (cinco profesores/a de la Universidad de Sevilla, tres de la Universidad Nacional de Educación a Distancia, dos de la Universidad de Huelva, tres de la Universidad de Castilla-La Mancha y cuatro de la Universidad Pablo de Olavide de Sevilla).

La población general o universo general en este estudio son todos los/as jóvenes andaluces entre 15 y 29 años, entendiendo por jóvenes andaluces a aquellos/as que se encuentran en el momento del estudio residiendo en la Comunidad Autónoma Andaluza.

Para determinar el tamaño de la muestra se utilizó la fórmula siguiente (Sierra Bravo, 1989):

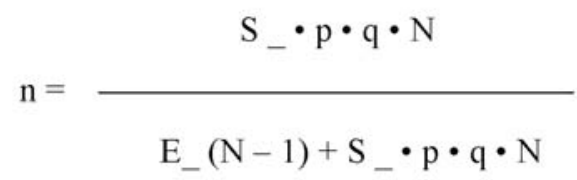

$\mathrm{n}=$ muestra

$\mathrm{S}$ = desviación típica, para el nivel de confianza elegido

$\mathrm{p}=\%$ de las características de estudio

$\mathrm{q}=100-\mathrm{p}$

$\mathrm{N}$ = población de estudio

$\mathrm{E}=$ error de estimación decidido

Para determinar el tamaño de la muestra, que resultó con un total de 2.066 jóvenes, establecimos un nivel de confianza del 95\%, se estimó un margen de error del $\pm 4 \%$ y un sistema de muestreo probabilístico y estratificado.

El cuestionario fue aplicado en las ocho provincias de la Comunidad Autónoma Andaluza, abarcando tanto zonas urbanas como rurales, y en poblaciones que superaban los 30.000 habitantes.

Una vez recogidos los datos se procedió a la codificación de los mismos. Es decir, se asignó un número a cada categoría de respuestas, el cual facilitará el proceso de reduc- 
ción de los datos para su correspondiente análisis. Este trabajo viene motivado por la necesidad de efectuar el tratamiento de los datos por ordenador, dado el volumen de los mismos y la rapidez con la que se puede llegar a trabajar.

El paquete que empleamos es el conocido por el paquete estadístico SPSS (Statistickal Package for Social Sciences).

\section{Análisis e interpretación de los resultados}

A continuación, presentamos una síntesis de los datos descriptivos globales del estudio señalando cuál es la tendencia que manifiestan los/as jóvenes andaluces encuestados.

\subsection{Diferencias entre la percepción que tienen los/as jóvenes andaluces del colectivo gitano y del colectivo de inmigrantes}

Con respecto al colectivo de gitanos, los/as jóvenes andaluces tienen una tendencia del $51,7 \%$ a manifestar que los gitanos son personas incultas e ignorantes, el 66,1\% manifiesta que son personas desconfiadas y mentirosas (48,6 \%). El 63,9\% considera que los gitanos constituyen un colectivo violento y peligroso.

Poco más de la mitad de los/as jóvenes andaluces, el 53,2\%, tienden a manifestar que los gitanos no se adaptan bien a la sociedad, viven independientes, alejados y excluidos de ella $(54,7 \%)$, sin respetar las normas sociales ni cumplir con sus deberes cívicos $(63,4 \%)$. En este sentido, el 65,5\% sostiene que los gitanos no están integrados en la sociedad y que no tienen interés por integrarse.

En cuanto al colectivo de inmigrantes, los/as jóvenes andaluces tienden a opinar en un $39 \%$ que son personas incultas e ignorantes, aunque el $44,2 \%$ manifiesta que son muy astutos y perspicaces. El 75,1\% considera a los inmigrantes como personas muy trabajadoras pero desconfiadas $(34,6 \%)$. Por otro lado, piensan que los inmigrantes son personas cordiales, amables, afables y de buen trato $(57 \%)$.

La mitad de los/as jóvenes que residen en Andalucía (53\%) opinan que los inmigrantes se adaptan bien a la sociedad, y el $72,7 \%$ de los encuestados declaran que los inmigrantes sí quieren integrarse y que buscan mejorar sus condiciones de vida $(72,2 \%)$.

Partiendo de los datos que arroja el cuestionario, podemos determinar que los/as jóvenes andaluces consideran que los inmigrantes son incultos, ignorantes y desconfiados; sin embargo, también los perciben como personas astutas e ingeniosas, muy trabajadores, sinceras, pacíficas, cordiales, amables, afables y de buen trato. Consideran que son personas que podrían adaptarse bien a la sociedad receptora, aunque viven independientes y alejados de ésta. Manifiestan que los colectivos de inmigrantes no están integrados en la sociedad, pero que desean integrarse buscando continuamente mejorar sus condiciones de vida; son personas saludables, buenos padres, y se interesan por la escuela y por la educación de sus hijos.

La percepción que tienen los/as jóvenes andaluces sobre las culturas en general es bastante positiva, si bien resulta algo inesperado comprobar cómo tienen una opinión más positiva de los inmigrantes que de los gitanos. Dato un tanto paradójico, dado que los gitanos vienen conviviendo en Andalucía desde hace varios siglos, mientras que los inmigrantes lo están haciendo desde fechas muy recientes.

Los inmigrantes, por el contrario, ofrecen más posibilidades de relación con la juventud, puesto que desempeñan actividades como la venta de artilugios musicales y discos compactos. Por otra parte, los medios de comunicación transmiten una imagen más cercana y asequible de los inmigrantes, que los/as jóvenes aceptan mejor. Las noticias 
que difunden los medios suelen estar relacionadas con las situaciones penosas por las que atraviesan los inmigrantes hasta llegar a establecerse en un país, estas circunstancias despiertan sentimientos favorables en el público en general, y en los/as jóvenes en particular.

\section{2. ¿Te molestaría tener que relacionarte con...?}

A raíz de la literatura consultada sobre el tema y basándonos en otras investigaciones, categorizamos diferentes colectivos tomando como referentes patrones culturales, patrones ideológicos, la raza, y diferentes zonas de procedencia, de tal forma que les preguntamos a los/as jóvenes andaluces si les molestaría mantener relaciones con estos colectivos.

La tendencia general es que a los/as jóvenes andaluces no les importaría mantener relaciones con gitanos, árabes, judíos, con personas de raza negra, asiáticos, latinoamericanos, norteamericanos, africanos y con europeos; sin embargo, destacamos la tendencia que existe en los/as jóvenes andaluces a manifestar que sí les molestaría mucho tener que relacionarse con personas del movimiento skin heads y ultrarradicales, así como con miembros terroristas; en relación a este último dato se observa que les molestaría más relacionarse con terroristas que con skin heads.

Sobre los colectivos con los que a los/as jóvenes andaluces no les molestaría tener que relacionarse, y haciendo referencia a los datos extraídos del cuestionario, al 90,8\% no le molesta relacionarse con latinoamericanos, al 89,4\% no le molesta relacionarse con asiáticos, a un $89,2 \%$ no le importa relacionarse con europeos, al 89,1\% no le importa tener que relacionarse con personas de raza negra, el 88,8\% manifiesta no molestarle relacionarse con norteamericanos y con africanos. En relación a los judíos, al 86,7\% de los/as jóvenes andaluces le es indiferente tener que relacionarse con ellos; por otro lado, al 80,5\% no les molestaría relacionarse con gitanos, y al 77,7\%, con árabes.

Como ya hemos comentado, la tendencia general manifiesta es que a los/as jóvenes andaluces no les molestaría tener que relacionarse con los distintos colectivos, lo que nos llevaría a pensar que estos jóvenes son tolerantes y respetuosos con personas procedentes de otro país y de distinta raza y/o cultura (exceptuando los colectivos que presentan rasgos violentos en su comportamiento como son los skin heads y terroristas). Sin embargo, nos resulta preocupante el dato de que el 22,3\% de los/as jóvenes andaluces manifiestan recelos a la hora de relacionarse con árabes, y que al 19,5\% le moleste relacionarse con gitanos.

\subsection{Si de ti dependiera, ¿a quién echarías de España?}

Uno de los apartados del cuestionario se centró en una serie de variables que hacían referencia a una pregunta clara y tajante. "Si de ti dependiera, ¿a quién echarías de España?". Formulada esta pregunta de esa forma, bastante prejuiciosa, pretendíamos saber qué porcentaje de jóvenes se manifestaban claramente etnocéntricos y racistas al desear "echar a los extraños".

Los datos extraídos de los cuestionarios revelan que la mayor parte de ellos, el 80,9\% de los/as jóvenes andaluces, tienden a manifestar que echarían de España a aquellas personas pertenecientes al movimiento skin heads. En relación a otros colectivos, la tendencia es que no echarían a ninguno de ellos, aunque destacamos que un número importante de jóvenes andaluces, el 24,6\%, echaría a los árabes, y un 14,9\% expulsaría a los gitanos. Resulta alarmante la tendencia que existe en algunos jóvenes al manifestar que si de ellos dependieran echarían de España a estos grupos. Son minoría, pero resulta preocupante la existencia de estos grupos de jóvenes que declaran su deseo de expulsar de España a los diferentes colectivos de personas. 
En líneas generales, los/as jóvenes andaluces se consideran tolerantes con otros colectivos y respetuosos con sus diferencias, principalmente los de 15 a 19 años, con estudios primarios y/o secundarios, que además residen en poblaciones pequeñas y medianas. Al respecto, conviene destacar que estos jóvenes son los que en sus respectivas poblaciones tienen menos ocasiones de contacto con grupos culturales diferentes, puesto que en estos lugares suele ser irrelevante la presencia de dichos grupos, por lo que estos resultados deberían tomarse con cierta precaución.

Por otro lado, los/as jóvenes andaluces que residen en grandes ciudades son los que en mayor medida han manifestado su deseo de expulsar a los grupos de cabezas rapadas del país. Tenemos que tener en cuenta que estos grupos fascistas suelen actuar en grandes ciudades, ya que en las pequeñas y medianas poblaciones su existencia es prácticamente nula.

\subsection{Conocimiento de los/as jóvenes andaluces sobre el fenómeno migratorio}

El conocer la valoración que hacen los/as jóvenes andaluces sobre determinados aspectos del fenómeno migratorio nos posibilita analizar la concepción que éstos tienen de una sociedad intercultural integrada y cohesionada. Por ello, un apartado central de este estudio se ocupó de determinar el conocimiento que tienen los/as jóvenes andaluces sobre el fenómeno migratorio y su repercusión en la sociedad en la que viven. Para ello se articularon las variables en torno a:

- La inmigración favorece la construcción de una nueva identidad cultural.

- Los inmigrantes deben abandonar su cultura para integrarse en la sociedad.

- Trabajo para los inmigrantes sólo si los autóctonos lo rechazan.

- Aportación de los inmigrantes a la Hacienda Pública. Utilización de los Servicios Sociales.
- Las políticas sociales y la Ley de Extranjería refuerzan el "efecto llamada".

En relación a si la llegada de inmigrantes posibilita la construcción de una nueva identidad cultural, existe una tendencia del 37,5\% a manifestar que sí favorece la construcción de una nueva identidad cultural, frente al $50,6 \%$ que se muestra contrario a ello. Por lo que la mitad de los/as jóvenes andaluces no creen que la llegada de inmigrantes favorezca la construcción de una nueva identidad cultural en la sociedad.

Centrándonos en la segunda cuestión, hay una clara tendencia en los/as jóvenes andaluces a manifestar que los inmigrantes nunca deben abandonar su cultura y asimilar la de la sociedad receptora, tendencia del $73,2 \%$. Por el contrario, el $18,7 \%$ sostiene que sí deben abandonar su cultura para insertarse en la sociedad.

El 63\% de los/as jóvenes andaluces manifiestan su total desacuerdo en que los inmigrantes deben cubrir un puesto de trabajo sólo cuando no haya españoles para desempeñarlo. Por otro lado, es alarmante que el $27 \%$ de estos jóvenes esté conforme con que exista una prioridad hacia las personas con nacionalidad española a la hora de acceder a un puesto de trabajo.

Cuando hemos preguntado si los inmigrantes aportan poco a la Hacienda Pública, pero en cambio se benefician de las políticas sociales, destaca que el 36,6\% de los jóvenes andaluces manifiestan un total desconocimiento al respecto. El $22 \%$ considera que aportan poco a Hacienda y se benefician mucho de las políticas sociales, y el 41,4\% se manifiesta contrario a esta idea.

En la variable sobre si realmente la política social y la actual Ley de Extranjería provocan un "efecto llamada" en los inmigrantes, destaca también el desconocimiento que hay sobre el tema $(38,3 \%)$. El 39,2\% de los/as jóvenes andaluces creen en el "efecto llamada" y consideran que la política social vigente y 
la Ley de Extranjería provocan este efecto aumentando la llegada de inmigrantes. Por el contrario, el 22,5\% no cree que la llegada de inmigrantes se deba a un "efecto llamada" por la situación social del país al que llegan.

En general, los/as jóvenes andaluces manifiestan que la llegada de inmigrantes a la sociedad no suele posibilitar la construcción de una nueva identidad cultural. Plantean que la identidad cultural de los inmigrantes no afecta a la identidad cultural nacional, la cual se mantendrá estática aunque se establezca un contacto con otras culturas. Este planteamiento podría ser el primer paso hacia un etnocentrismo cultural por parte de los/as jóvenes andaluces.

La juventud andaluza se manifiesta en contra de la asimilación cultural como medida de integración, al sostener que los inmigrantes no deben abandonar su cultura y asimilar la de la sociedad receptora.

$\mathrm{Si}$ anteriormente hemos concluido que estos jóvenes no creen en la posibilidad de una cultura única integrada por todos los $\mathrm{co}^{-}$ lectivos, y se manifiestan en contra de que los inmigrantes abandonen su cultura y asimilen la cultura dominante, ¿por qué modelo de integración apuestan los/as jóvenes andaluces? Los jóvenes andaluces apuestan por una integración que respete los derechos culturales de cada colectivo, pero sin que la cultural mayoritaria se vea influenciada por las culturas minoritarias, sino más bien al contrario, es decir, manifiestan un respeto por los diferentes colectivos culturales siempre y cuando se acomoden a las formas de vida de la sociedad dominante.

Otro aspecto que planteamos fue si en la concesión de un empleo debe primar la nacionalidad española o, por el contrario, todas las personas deben tener las mismas oportunidades. En líneas generales, se observa un talante democrático en los/as jóvenes andaluces al manifestar que todas las personas deben tener el mismo trato y las mismas oportunidades para acceder a un puesto de trabajo, si bien debemos señalar que el $27 \%$, más de un cuarto de la población joven andaluza, opina que los inmigrantes deben realizar sólo los trabajos que no desean los españoles.

Sobre la aportación que realizan los inmigrantes a la sociedad de acogida, un alto porcentaje de los/as jóvenes andaluces desconoce la aportación económica que realizan los inmigrantes, así lo manifiesta el $36,6 \%$ de los encuestados. La mayoría de éstos (el 41,3\%) expresa que los inmigrantes aportan poco al desarrollo económico del país y, por el contrario, se benefician de los servicios sociales. Las jóvenes andaluzas son las que en mayor medida han manifestado esta idea. Estos datos contrastan con una realidad muy diferente, ya que el trabajo de los inmigrantes repercute de forma positiva en el crecimiento económico del país, así como en su demografía.

Un porcentaje considerable de jóvenes andaluces $(39,2 \%)$ cree en el "efecto llamada", es decir, en que las condiciones sociales y la protección jurídica que ofrece el Gobierno español provocan un efecto de atracción incrementando el número de inmigrantes que llegan de forma legal e ilegal. Por otro lado, encontramos a un grupo numeroso de jóvenes que manifiesta desconocimiento al respecto $(38,3 \%)$.

En este sentido, los jóvenes que residen en Cádiz, Granada, Málaga y Sevilla tienden a manifestar que los inmigrantes llegan a España atraídos por las condiciones sociales y económicas del país. Por el contrario, aquellos que residen en Almería, Huelva y Jaén, ciudades que cuentan con mayor población inmigrante que el resto, son los que en menor medida creen en la existencia del denominado "efecto llamada".

\subsection{Requisitos para la integración de los inmigrantes}

En lo referente a qué debe de adquirir una persona inmigrante para integrarse en la socie- 
dad a la que llega, destacamos la tendencia que hay entre los/as jóvenes andaluces a manifestar que, en primer lugar, un inmigrante debe acceder legalmente al país; en segundo lugar, tener su documentación en regla, y en tercer lugar, conocer el idioma del país. La misma tendencia nos encontramos al situar en cuarto y en quinto lugar la consecución de un empleo y tener una formación profesional. Por último, sitúan asimilar la cultura de la sociedad en la que quieren insertarse.

En este sentido, los sujetos estudiados prefieren que los inmigrantes se integren en nuestra sociedad, aunque existen algunos matices diferenciales que conviene señalar en relación a la integración de extranjeros. Así, para los varones de más edad (de 25 a 29 años) diplomados o licenciados, que trabajan y residen en pequeñas y medianas ciudades, el factor que más incide en la integración efectiva de los extranjeros es que jurídicamente tengan una situación de legalidad y posean su documentación y los permisos en regla y vigentes. De todos estos jóvenes, esta respuesta se da, sobre todo, entre aquellos que residen en Sevilla.

Sin embargo, las jóvenes andaluzas de menor edad (de 15 a 19 años), con estudios primarios o secundarios, que se encuentran estudiando, tienden en mayor medida a establecer como elementos importantes para la integración de los extranjeros el aprendizaje de la lengua, la inserción laboral y el conocimiento de la cultura y las costumbres de la sociedad de acogida. Es preciso señalar que de estas jóvenes, aquellas que residen en Almería son las que en mayor proporción dan importancia a la inserción laboral de los inmigrantes como una de las medidas de integración social.

\subsection{En síntesis}

A continuación presentamos una valoración de los datos obtenidos sobre la percepción que tienen los/as jóvenes andaluces de los inmigrantes y las minorías culturales, con objeto de dictaminar las propuestas de acción que consideramos oportunas, y así avanzar en un modelo de sociedad interculturalmente cohesionada.

El presente estudio ha puesto de manifiesto cómo los/as jóvenes andaluces expresan opiniones diferentes hacia los gitanos con respecto a los inmigrantes. Tienen una percepción de los gitanos muy negativa, al contrario de lo que sucede con los inmigrantes, de tal forma que los/as jóvenes andaluces opinan que este colectivo se adapta mejor que los gitanos a la sociedad, son más trabajadores, de mejor trato y más pacíficos; sin embargo, sostienen que los gitanos no se adaptan a la sociedad viven alejados de ella, son vagos, intratables y muy violentos.

En relación a la diferencia que se establece entre la percepción que tienen sobre los gitanos y la que tienen sobre los inmigrantes, ésta se fundamenta en que, aunque los gitanos son ciudadanos del país, éstos han permanecido fuertemente cohesionados como grupo cultural, manteniendo sus costumbres y tradiciones. No se ha establecido una convivencia entre la sociedad dominante y este grupo social; por el contrario, han permanecido segregados y, en la mayoría de las ocasiones, marginados. Los valores presentes en la sociedad dominante no se corresponden con los valores culturales de este grupo étnico, por lo que siempre han preservado sus costumbres y tradiciones.

Los inmigrantes, por el contrario, tienen un mayor intercambio con la sociedad y con la juventud, puesto que buscan principalmente una inserción social y laboral que les permita desarrollarse como individuos, si bien, tal y como ya hemos señalado, se está experimentando una proliferación de grupos de inmigrantes en función del país de origen, favoreciendo la creación de guetos de estos colectivos en muchos barrios de las grandes ciudades. 
Muy pocos de los/as jóvenes andaluces se declaran racistas o xenófobos. Incluso cuando las preguntas son indirectas (trabajar junto con..., establecer relaciones íntimas con...), tampoco se aprecian actitudes intensas de rechazo. Manifiestan ser tolerantes con todos los colectivos que pero se aprecia que las personas por las que los entrevistados sienten más simpatía, son más deseadas como amigos, compañeros de trabajo o pareja son los latinoamericanos, asiáticos, europeos -occidentales-y los menos deseados, los árabes y después los gitanos. Pero en estas cuestiones se observa que los niveles de discriminación van aumentando en comparación con los resultados que se obtuvieron de otros estudios sobre los jóvenes madrileños en 1999 (Calvo Buezas, 2001).

En el estudio se constatan los alarmantes porcentajes de aquellos jóvenes que abiertamente han manifestado su rechazo hacia los: latinoamericanos, asiáticos, europeos, negros, norteamericanos, africanos, judíos, gitanos y árabes.

La existencia de un grupo minoritario racista y xenófobo se pone de manifiesto en aquellos jóvenes que proclaman abiertamente la expulsión del país de los judíos $(11,4 \%)$, gitanos $(14,9 \%)$ y árabes $(24,6 \%)$. El porcentaje de personas que clara y manifiestamente se pronuncian de forma tan etnocéntrica y racista de "echar a los extraños" decrece entre 1998 y 2003 (Calvo Buezas, 2001) en relación a los gitanos y los árabes; en este sentido, casi un cuarto de la juventud andaluza se declara abiertamente racista en relación a los árabes.

En relación a los jóvenes andaluces, el presente estudio desvela una serie de aspectos que no podemos perder de vista: el aumento de los prejuicios en los/as jóvenes hacia determinados colectivos; la aceptación por parte de estos jóvenes de determinados grupos racistas y violentos, y el incremento del racismo y la xenofobia entre la juventud.
En la sociedad está latente un racismo que si bien aún no es sentido por la sociedad, se pone de manifiesto a través de determinadas acciones violentas contra diversos colectivos (inmigrantes, gitanos, vagabundos, etc.), mostrándonos la existencia de la intolerancia y el etnocentrismo que se están potenciando en la sociedad.

La juventud andaluza se posiciona en contra de la asimilación cultural como medida de integración, al sostener que los inmigrantes no deben abandonar su cultura y asimilar la de la sociedad receptora. Sin embargo, se está produciendo un aumento de los/as jóvenes que muestran una actitud de cerrado etnocentrismo y de falta de apertura a otros pueblos y culturas.

También se observa un talante democrático en los/as jóvenes andaluces al considerar que todas las personas deben tener el mismo trato y las mismas oportunidades para acceder a un puesto de trabajo, si bien debemos señalar que el $27 \%$ (más de un cuarto de la población joven andaluza) opina que los inmigrantes deben realizar sólo los trabajos que no desean los españoles.

Sobre el conocimiento que tienen los/as jóvenes andaluces del fenómeno migratorio y de sus consecuencias económico-sociales, un alto porcentaje de los/as jóvenes andaluces desconocen la aportación económica que realizan los inmigrantes al país, así lo manifiesta el 36,6\% de los encuestados. La mayoría de éstos (el 41,3\%) tiende a manifestar que los inmigrantes aportan poco al desarrollo económico del país y, por el contrario, se benefician de las prestaciones sociales. Estos datos contrastan con una realidad muy diferente de lo que opinan los jóvenes, ya que parte del desarrollo económico del país se debe al trabajo realizado por este colectivo. Por otro lado, el sistema de bienestar social y la protección jurídica que el país les ofrece favorecen la llegada de los inmigrantes, esta idea la manifiestan la mayor parte de los/as jóvenes andaluces. 


\section{Conclusiones}

En todos los estudios consultados sobre la temática, muy pocas personas se declaran racistas o xenófobas, incluso cuando las preguntas son indirectas. Tampoco se aprecian actitudes intensas de rechazo, aunque se observan diferencias según el origen. En este sentido, como nos indica Calvo Buezas (2001), son pocos los racistas militantes, pero altamente peligrosos: hacen daño a pocos, a muy pocos, pero atemorizan a muchos. Sin embargo, nos señala este autor, son mucho más los/as jóvenes solidarios que el grupito racista.

Según el citado autor, el prejuicio discriminatorio contra los extranjeros tiene una base principal de tipo socioeconómico más que racial o cultural, salvo en el caso de los gitanos. Este autor plantea que el prejuicio discriminatorio está fundamentado por la condición de marginal que presentan muchos inmigrantes debido a su situación laboral, a la carencia de recursos, a situaciones legales irregulares, al hecho de tener la residencia en áreas urbanas marginales y excluidas, etc. En este sentido, también planteamos que el prejuicio xenófobo crece conforme se baja de posición social o educativa y según el grado de marginalidad que presente el inmigrante en sí, no discriminando ni rechazando la gran mayoría de las personas a una cultura o una raza en sí, salvo como hemos apuntado en el caso de los gitanos.

En una sociedad global, la diversidad no es un obstáculo, sino más bien un requisito para el desarrollo personal y social. La cohesión social y el compromiso de participar en la vida de la sociedad se refuerzan cuando se reconocen y valoran las diferencias. En la sociedad se están desarrollando espacios cada vez más multiétnicos, pluriculturales y mestizos. Si los/as jóvenes de ahora no aprenden a convivir juntos en las diferencias, es previsible el auge del racismo y de la xenofobia, recrudeciéndose aún más los conflictos interétnicos.
Una verdadera democracia es el respeto a las minorías, sean éstas políticas, ideológicas, religiosas, étnicas o culturales. De ahí la necesidad de educar a todos los/as jóvenes en los valores democráticos de la solidaridad y del respeto a las minorías, a los "diferentes", a los "otros". Y hay que confiar en los/as jóvenes. El camino de la tolerancia y del pluralismo cultural, y por ende de una auténtica democracia, es largo, difícil y complejo. El conocer esos valores, así como esos prejuicios xenófobos y racistas, fue el objetivo de esta investigación. "Conocer para prever, prever para poder".

Por otro lado, planteamos que para poder desarrollar una sociedad integrada y cohesionada, tanto los inmigrantes como las minorías étnicas deben conocer el medio social en el que se desenvuelven, deben aprender a manejar los elementos que les permitan desenvolverse en él de manera autónoma. Pero la integración es algo que nos afecta a todos, y es también responsabilidad de la sociedad de llegada fomentar el desarrollo de acciones integradas a través de procesos interculturales. Para ello creemos necesaria la creación de espacios de encuentros donde las personas puedan interaccionar y potenciar el acercamiento y el conocimiento de otras culturas y de otras formas de pensar; sólo así podremos apostar por una sociedad intercultural, avanzando en la creación de una nueva identidad social donde tenga cabida toda la diversidad presente en las sociedades democráticas.

La apuesta por una gestión intercultural implica rechazar prejuicios y respetar la diversidad, además de aceptar y apreciar el mutuo reconocimiento de todas las culturas. Para ello es necesario conocer la diversidad, valorarla y preservarla, así como comprometer a todos, a minorías y mayorías autóctonas.

Apostamos por el reconocimiento intercultural de la diferencia, construyendo un diálogo igualitario entre culturas y un pro- 
yecto común de convivencia democrática, donde los derechos alcancen a todos y donde los deberes cívicos también sean exigibles a todos. A su vez, creemos que el reconocimiento intercultural de la igualdad no debe reclamar a los inmigrantes y a las minorías que renuncien a sus identidades culturales, buscando la asimilación o segregación xenófoba; al contrario, debe buscar, desde el respeto a su identidad, afirmar los derechos humanos y la dignidad como valores universales.

Debemos configurar políticas concretas de lucha contra el racismo, acciones en el campo de la educación y sensibilización, singularmente en la escuela y medios de comunicación social, incluyendo las nuevas tecnologías, con la prevención del odio racial o étnico. Es necesario elaborar políticas que impliquen a los/as jóvenes en el desarrollo de la propia sociedad en la que viven y a través de ésta, en el desarrollo de la sociedad mundial. Fomentar políticas integrales, dirigidas tanto a integrar a la juventud en la dinámica de las instituciones sociales como a desarrollar cauces de participación real, responsable y comprometida de los jóvenes. Tal y como nos señala Froufe (1999), "la integración como modelo consiste en mantener la identidad de cada cultura y en valorar de una forma positiva las relaciones entre ellas. La integración favorece el interculturalismo siempre que se interprete como la integración de unos con otros, y no como un mero integrarse en un espacio social" (pp. 16).

Hemos de educar en los principios de convivencia, comprensión, generosidad, honradez, trabajo, profesionalidad. En muchas ocasiones se piensa que educar en valores es cuestión de niños y adolescentes, como si los adultos no lo necesitaran. Todos necesitamos, hasta el final de nuestra existencia, educarnos continuamente en actitudes y comportamientos de apertura y comprensión hacia los otros, particular- mente hacia los otros que consideramos "más extraños y diferentes".

Nuestra función es combatir los fáciles estereotipos y clarificar que no se trata propiamente de luchas de civilizaciones, ni de luchas de religiones, pueblos y culturas. El desafío es enseñar y aprender a convivir en la diferencia, cumpliendo con los derechos humanos fundamentales y con los valores de la igualdad, la justicia y la solidaridad. Y estos valores tenemos que aprenderlos: no nacemos terroristas ni guerreros, pero tampoco pacíficos y demócratas. Hay que pasar de una cultura de la guerra a una cultura de la paz. Y en esa tarea, en esa educación de valores pacifistas y solidarios, también la Universidad tiene mucho que decir y que hacer.

Tal y como nos indica Rodríguez Izquierdo (2007), "la educación social debe promover y garantizar las competencias fundamentales para una sana convivencia y para el ejercicio de la ciudadanía responsable", porque no nacemos racistas ni fascistas, pero tampoco demócratas y solidarios: nos hacemos a lo largo de toda nuestra andadura existencial.

\section{Referencias bibliográficas}

Calvo Buezas, T. (2001): Inmigración y Universidad. Prejuicios racistas y valores solidarios. Madrid: Editorial Complutense

Froufe Quintas, S. (1999): "Educación intercultural y pedagogía de la interculturalidad”. Pedagogía Social. Revista Interuniversitaria, 3, pp. 9-26.

Mateos Claros, F. (1995): Un modelo de evaluación institucional: coste/eficacia del centro asociado de Algeciras. Sevilla: INATED.

Pérez de Guzmán Puya, V. (2003): “La investigaciónacción como punto de partida para trabajar con inmigrantes", en AA VV: Educación ocial $e$ inmigración. Sevilla. Sociedad Iberoamericana de Pedagogía Social.

Pérez Serrano, G. (2002): Juventud: estudio socioeducativo. Sevilla: Universidad de Sevilla. 
Rodríguez Izquierdo, R. (2007): “Contradicciones y desafíos de la globalización para la Educación Social". Pedagogía Social. Revista Interuniversitaria, 14, pp. 119-127.

\section{Notas}

${ }^{1}$ Referencia de la investigación: 2003.Partida presupuestaria de la financiación por parte del Instituto Andaluz de la Juventud.
DiRECCIÓN DE LA AUTORA: María del Rocío Cárdenas Rodríguez. Universidad Pablo de Olavide. Departamento de Ciencias Sociales. Carretera de Utrera, km. 1. 41013 Sevilla. Correo electrónico:mrcarrod@upo.es

Fecha de recepción del artículo: 22.VII.2007 Fecha de aceptación definitiva: 05.IX.2007 